\title{
Clinical Profile of Atypical Manifestations of Dengue Fever
}

\author{
Sriram Pothapregada $^{1} \cdot$ Banupriya Kamalakannan $^{1} \cdot$ Mahalakshmy Thulasingam $^{2,3}$
}

Received: 21 April 2015 / Accepted: 26 October 2015 / Published online: 4 January 2016

(C) Dr. K C Chaudhuri Foundation 2015

\begin{abstract}
Objectives To study the clinical profile and outcome of the atypical manifestations of dengue fever in children.

Methods All children (0-12 y of age) diagnosed and confirmed as dengue fever at a tertiary care hospital at Puducherry, between the 1st of August 2012 and January 31st 2015 were reviewed retrospectively from hospital case records as per the revised World Health Organization (WHO) guidelines 2011 for dengue fever. The diagnosis was confirmed by NS1 antigenbased ELISA test or dengue serology for IgM and IgG antibodies and the data was analyzed using SPSS 16.0 statistical software.

Results Out of 254 children admitted with dengue fever, nonsevere dengue and severe dengue were seen in $62.6 \%$ and $37.4 \%$ respectively. Atypical manifestations were seen in 106 cases $(41.7 \%)$. Mean age of presentation was 6.9(3.3) y. M: F ratio was 1.2:1. The common manifestations of severe dengue infection were shock $(37.4 \%)$, bleeding $(20.1 \%)$ and multiorgan dysfunction $(2.4 \%)$. The most common atypical manifestations of dengue fever were lymphadenopathy $(41.7 \%)$, splenomegaly $(21.2 \%)$, biphasic fever $(18.1 \%)$, hepatitis $(11.4 \%)$, febrile diarrhea (6.3\%), refractory shock (2.4\%) and impaired consciousness (1.9\%). The other atypical manifestations present were portal hypertension, acalculous
\end{abstract}

Sriram Pothapregada

psriram_ped@yahoo.co.in

1 Department of Pediatrics, Indira Gandhi Medical College and Research Institute, Kathirkamam, Puducherry 605009, India

2 Department of Community Medicine, Jawaharlal Institute of Postgraduate Medical Education and Research, Puducherry, India

3 Department of Community Medicine, Indira Gandhi Medical College and Research Institute, Kathirkamam, Puducherry, India cholecystitis, appendicitis, acute respiratory distress syndrome (ARDS), myocarditis, pericardial effusion, paroxysmal supraventricular tachycardia (PSVT), myositis, acute kidney injury (AKI), hemophagocytic syndrome and disseminated intravascular coagulopathy (DIC). Platelet count did not always correlate well with the severity of bleeding. There were six deaths (2.4\%) and out of them four presented with impaired consciousness $(66.6 \%)$. The common causes for poor outcome were multiorgan failure, encephalopathy and refractory shock. Conclusions The atypical manifestations of dengue fever are no more a rare entity. Clinicians should have a high index of suspicion and vigilance for atypical manifestations of dengue fever as lack of timely detection and management could be fatal. Impaired consciousness was the most ominous atypical manifestation of severe dengue infection.

Keywords Dengue fever · Encephalopathy · Myositis · Atypical manifestations $\cdot$ Hepatitis $\cdot$ Myocarditis

\section{Introduction}

Dengue fever is the most rapidly spreading mosquito borne viral disease worldwide with an estimated 30-fold increase in incidence over last five decades [1]. With rising disease burden, atypical manifestations are also on rise, but are underreported and often missed due to the lack of awareness among health care personnels. These include neurological, hepatic, renal, cardiovascular and other isolated organ involvement and termed as Expanded dengue syndrome/unusual or atypical manifestations of dengue fever as per the revised 2011 guidelines of dengue fever and are often life threatening with very high case fatality rate [1]. Atypical manifestations could be explained as complications of severe profound shock, associated underlying host conditions, diseases and co-infections [2, 3]. They might 
resemble co-infections like enteric fever and malaria thereby making the clinical decision more difficult and mislead the physician's initial impression. There is limited literature describing atypical manifestations of dengue fever in children. In this communication, the authors describe the atypical manifestations in pediatric in-patients with serologically confirmed dengue virus infection.

\section{Material and Methods}

After approval by the Institute Ethics committee, case records of all children (0-12 y of age) diagnosed and confirmed as dengue fever at a tertiary care hospital at Puducherry between the 1st August 2012 and January 31st 2015 were reviewed retrospectively from the hospital case records. Data was entered in a structured proforma. The case definition, diagnosis and management used for dengue fever, severe dengue and atypical manifestations were as per the revised World Health Organization (WHO) guidelines 2011. The diagnosis was confirmed by NS1antigen-based ELISA test (J. Mitra kit, India) or dengue serology for IgM and IgG antibodies (Kit from National Vector Born Disease Control Programme, Pondicherry and National Institute of Virology, Pune, India). All other relevant and other additional investigations were done as per the clinical course of illness.

The SPSS 16.0 statistical software was used for data analysis. Categorical variables were expressed as frequencies and percentages, and then analyzed by the $\chi^{2}$ test or fishers exact test, where appropriate. Continuous data, expressed as mean $\pm \mathrm{SD}$, or median (range), were analyzed using students t-test, analysis of variance (ANOVA-1 way) or Mann-Whitney U test. Odds ratio (OR) with $95 \%$ confidence interval (CI) was calculated to measure the degree of association of warning signs with severe dengue infection. Significance was taken at $P$ value $<0.05$.

\section{Results}

Out of 254 children admitted with dengue fever, non-severe dengue and severe dengue were seen in $62.6 \%$ and $37.4 \%$ respectively. Atypical manifestations were seen in 106 cases $(41.7 \%)$ and among them severe complications with multiorgan involvement were seen in 16 cases $(6.3 \%)$. The most common affected age group was 6-12 y (58.2\%) and the mean age of presentation was 6.9(3.3) y. Male to female ratio was 1.2:1. Most cases were from Pondicherry and its neighboring states.

Fever duration of $<7 \mathrm{~d}$ was present in 185 cases $(72.8 \%)$, $>7 \mathrm{~d}$ in 23 cases $(9 \%)$ and biphasic fever in 46 cases $(18.1 \%)$. The most common clinical warning signs at admission were persistent vomiting (77.1\%), liver enlargement (62.9\%), cold and calmy extremities (42.5\%), pain abdomen (31.8\%), hypotension $(31.4 \%)$, restlessness $(27.1 \%)$, giddiness $(23.6 \%)$, bleeding $(20.1 \%)$, oliguria $(12.9 \%)$, lethargy (6.3\%), pleural effusion (5.1\%), and impaired consciousness with Glasgow coma scale $(\mathrm{GCS})<8(1.9 \%)$.

The most common manifestations of severe dengue infection were shock $(37.4 \%)$, bleeding $(20.1 \%)$ and multiorgan failure $(2.3 \%)$. Ninety five children with severe dengue (78.8\%) had compensated shock and only $32.3 \%$ of them had bleeding. Atypical manifestations were seen in 106 $(41.7 \%)$ children (Table 1$)$.

Neurological manifestations were seen in 28 children $(11 \%)$ and among them impaired consciousness at admission $(\mathrm{GCS}<8)$ was seen in 7 cases, seizures in 17 children, two children had intracranial hemorrhage, aseptic meningitis in 2 cases and one child had pyogenic meningitis.

Hepatic dysfunction was seen in 29 children (11.4\%) and among them 16 children had features of shock. Fulminant hepatic failure was present in two cases. Two children had

Table 1 Atypical manifestation of dengue fever

\begin{tabular}{ll}
\hline Atypical manifestations & Number (\%) \\
\hline Seizures & $17(6.7)$ \\
Encephalopathy & $7(2.7)$ \\
Intracranial hemorrhage & $2(0.8)$ \\
Hepatitis & $29(11.4)$ \\
Fulminant hepatic failure & $2(0.8)$ \\
Acalculous cholecystitis & $2(0.8)$ \\
Acute pancreatitis & $1(0.4)$ \\
Acute parotitis & $2(0.8)$ \\
Acute kidney injury & $6(2.4)$ \\
Myocarditis & $5(1.9)$ \\
Pericardial effusion & $3(1.2)$ \\
Paroxysmal supraventricular tachycardia & $3(1.2)$ \\
Sinus bradycardia & $2(0.8)$ \\
Lymphadenopathy & $106(41.7)$ \\
Appendicitis & $2(0.8)$ \\
Pulmonary hemorrhage & $4(1.6)$ \\
Acute respiratory distress syndrome & $4(1.6)$ \\
Pleural effusion & $13(5.1)$ \\
Ascites & $18(7.0)$ \\
Pneumonia & $31(12.2)$ \\
Hemophagocytic syndrome & $2(0.8)$ \\
Splenomegaly & $54(21.2)$ \\
Refractory shock & $6(2.4)$ \\
Biphasic fever & $46(18.1)$ \\
Febrile diarrhea & $16(6.3)$ \\
Myositis & $3(1.2)$ \\
Disseminated intravascular coagulopathy & $4(1.6)$ \\
Co-infection & $19(7.5)$ \\
\hline &
\end{tabular}

Data as Number (\%) 
acalculous cholecystitis, two children had appendicitis, one child presented as acute pancreatitis and two children had portal hypertension.

The cardiovascular complication encountered was compensated shock and was present in 95 cases $(37.4 \%)$, decompensated shock was present in 16 cases $(6.3 \%)$ and fluid refractory shock in 6 children (2.4\%). Five children had myocarditis and among them global hypokinesia was seen in 3 children and diastolic dysfunction was seen in 2 children and received inotropic support. Three children had mild pericardial effusion without tamponade. Repeat echocardiographic evaluation at the time of discharge was normal in all the cases. Among conduction abnormality of the heart, sinus bradycardia was seen in two children and paroxysmal supraventricular tachycardia was seen in 3 children. One child had fluid refractory shock with unstable supraventricular tachycardia (SVT) which reverted with adenosine and the patient's circulatory status normalized. Among sixteen children who had decompensated shock, six children developed fluid refractory shock not responding to crystalloid and colloids and received inotropic support. Apart from shock, third spacing were seen in the form of pleural effusion in 13 cases $(50.4 \%)$, ascites in $18(46.7 \%)$ cases, and anasarca in 3 cases $(1.2 \%)$. The common respiratory complications like pneumonia was seen in 31 cases $(12.2 \%)$ and ARDS were seen in 4 cases $(2.4 \%)$. Acute kidney injury was seen 6 children with oligo-anuria and a mean creatinine value of $2.1 \pm 0.6 \mathrm{mg} / \mathrm{dl}$, and among them two children required peritoneal dialysis.

Spontaneous bleeding was present in 51 cases $(20.1 \%)$ and melena was the most common form of spontaneous bleeding. Tourniquet test was positive in 33 cases $(12.9 \%)$. Disseminated intravascular coagulopathy was seen in 4 children. The other atypical manifestations seen were febrile
Table 2 Laboratory parameters in dengue fever

\begin{tabular}{ll}
\hline Laboratory parameters & Number $(\%)$ \\
\hline Anemia $($ Hemoglobin $<10 \mathrm{~g} / \mathrm{dl})$ & $77(30.3)$ \\
Leukopenia $\left(\mathrm{TLC}<4000 / \mathrm{mm}^{3}\right)$ & $50(19.7)$ \\
Thrombocytopenia $\left(<1,50,000 / \mathrm{mm}^{3}\right)$ & $204(80.3)$ \\
PLC $<50,000 / \mathrm{mm}^{3}$ & $43(16.9)$ \\
PLC $<20,000 / \mathrm{mm}^{3}$ & $12(4.7)$ \\
Hemoconcentration $(\mathrm{HCT}>40)$ & $121(47.6)$ \\
Prothrombin time $(\mathrm{PT} / \mathrm{INR}>1.0)$ & $9(3.5)$ \\
APTT $(>1.5$ times normal) & $4(1.6)$ \\
Abnormal LFT (SGOT \& SGPT $>150 \mathrm{IU})$ & $29(11.4)$ \\
Deranged RFT (Serum creatinine $>3 \mathrm{mg} / \mathrm{dl})$ & $6(2.4)$ \\
Hypoalbuminemia (serum albumin $<3 \mathrm{~g} / \mathrm{dl})$ & $19(7.5)$ \\
Hypocalcemia (serum calcium $<9 \mathrm{mg} / \mathrm{dl})$ & $22(8.6)$ \\
Hyponatremia $($ Sodium $<130 \mathrm{Meq} / \mathrm{L})$ & $14(5.5)$ \\
Gall bladder wall edema on USG & $25(9.8)$ \\
NS1 antigen positive & $214(84.2)$ \\
NS1 antigen -ve and IgM positive & $40(15.7)$ \\
Dengue IgM positive & $140(55.1)$ \\
Dengue IgG antibody & $40(15.7)$ \\
\hline
\end{tabular}

PLC Platelet count; APTT Activated partial thromboplastin time.

diarrhea, biphasic fever, splenomegaly, generalized lymphadenopathy, hemophagocytic syndrome, myositis, and parotitis (Table 1). Co-infections were present in 19 cases (10 children had enteric fever, four had malaria, 4 had urinary tract infection and one had bacterial meningitis).

The hematological parameters showed anemia (30.3\%), leukopenia (19.7 \%) and thrombocytopenia in 204(80.3\%) cases (Table 2). Severe thrombocytopenia was seen in 43 cases $(16.9 \%)$, hemoconcentration in $47.6 \%$ of cases and
Fig. 1 Relationship between thrombocytopenia, bleeding and platelet transfusion

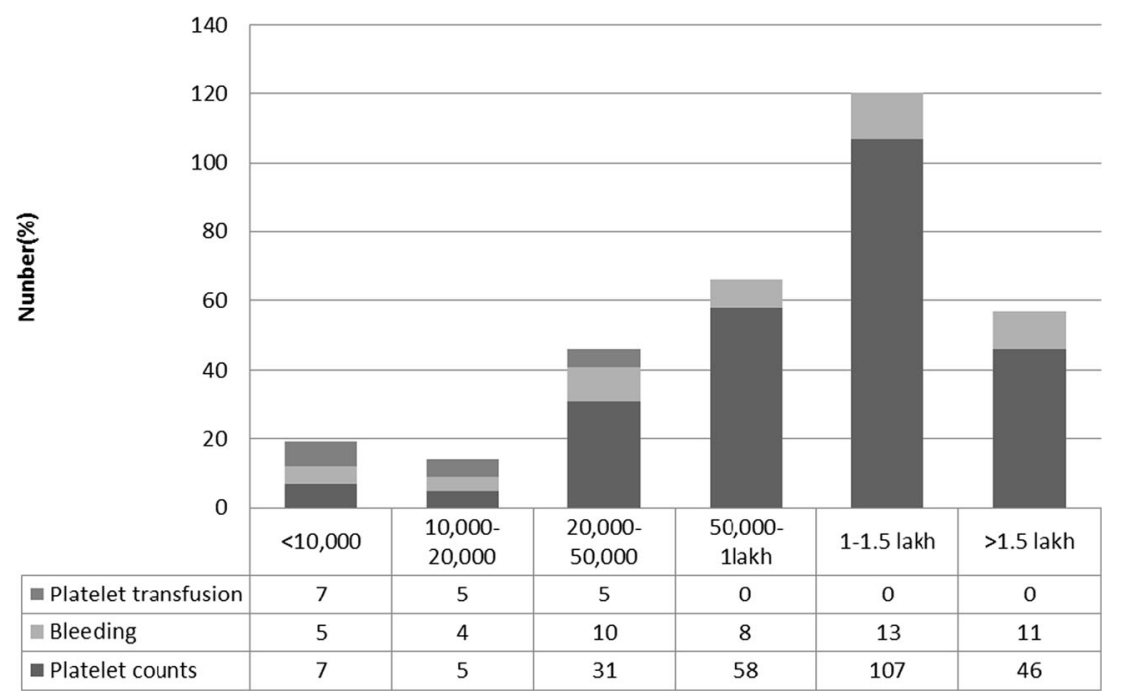


the mean haematocrit was 38.9(4.4). NS1 antigen was positive in 214 cases $(84.2 \%)$ and dengue $\operatorname{IgG}$ antibody was positive in 40 cases $(15.7 \%)$. Disordered coagulation (prolongation of the prothrombin and/or activated partial thromboplastin time) was seen in 13 children (5.1\%). Altered liver enzymes were seen in $11.4 \%$ of cases. Ultrasonography of abdomen was done in 105 children with severe dengue, and it was abnormal in 30 cases (16 hypoechoic hepatic parenchyma, 18 with ascites, 13 with pleural effusion, 25 with peri-gallbladder edema with wall thickening, 2 having cholelithiasis in addition, and 2 showing portal cavernoma with portal vein thrombosis). The CSF was abnormal in three patients, 2 of whom had raised protein and lymphocytic pleocytosis and one child showed evidence of bacterial meningitis. CT scan showed intracranial bleed in 2 children.

Figure 1 shows relationship between thrombocytopenia, bleeding and platelet transfusion. $78.4 \%$ of children with spontaneous bleeding had thrombocytopenia and among them $27.5 \%$ had features of shock. Bleeding manifestation was observed in $75 \%$ and $32.2 \%$ children with platelet counts of $<20,000$ and $20,000-50,000 / \mathrm{mm}^{3}$ respectively. $23.9 \%$ of cases with normal platelet count had bleeding and did not require platelet transfusion. Only 17 children $(6.7 \%)$ required platelet transfusions and out of them, $70.5 \%$ had a platelet count $<20,000 / \mathrm{mm}^{3}$ whereas $29.5 \%$ had platelet count in the range of $20,000-50,000 / \mathrm{mm}^{3}$. Only those children with significant spontaneous bleed or shock or with severe thrombocytopenia necessitated platelet transfusion.

Ninety six children $(37.7 \%)$ required intravenous fluids and blood component therapy was used in $12.9 \%$ cases. Seventeen children $(6.3 \%)$ required platelet transfusion, 6 children $(2.3 \%)$ required fresh frozen plasma, 10 children $(3.9 \%)$ required packed cell transfusion, 3 cases required colloids $(1.1 \%)$ and the requirement was more in severe dengue infection. Though not indicated as per WHO guidelines, all patients with count less than 20,000 received platelet transfusion while $16.1 \%$ of patients between $20,000-50,000 / \mathrm{mm}^{3}$ were transfused due to bleeding manifestations. However, platelets were not transfused with platelet counts $>50,000 /$ $\mathrm{mm}^{3}$ even in the presence of bleeding. Ten children required ionotropic support and mechanical ventilation.

The warning signs which were commonly associated with expanded dengue syndrome were prolonged shock, bleeding, pain abdomen, lack of clinical improvement post defervescence, impaired consciousness, lethargy, restlessness and severe thrombocytopenia (Fig. 2).

There were six deaths $(2.4 \%)$ and out of them four presented with impaired consciousness $(66.6 \%)$ at admission with GCS $<8$. The common causes for poor outcome were multiorgan failure, encephalopathy and refractory shock. The mean duration of hospital stay was $8.1(2.3) \mathrm{d}$ and the mean time to death was $2.7(2.3) \mathrm{d}$. The average period of recovery was longer in patients with severe dengue

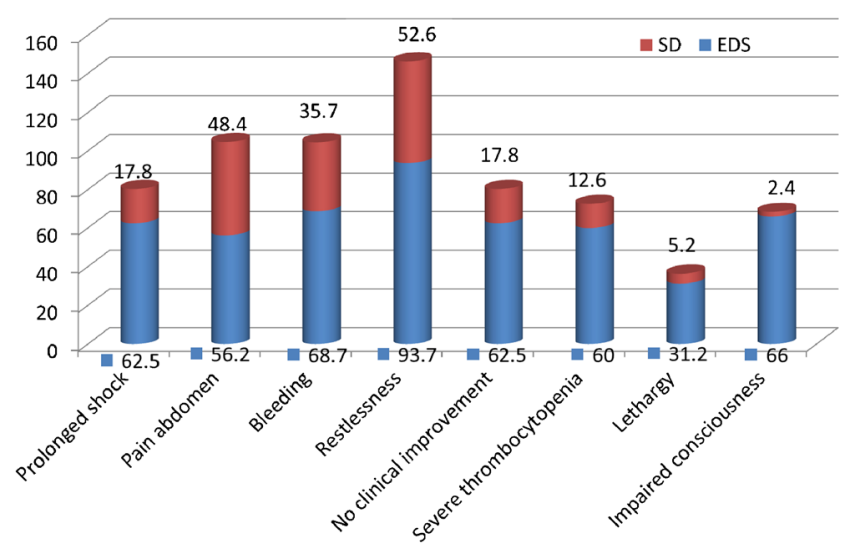

Fig. 2 Warning signs associated with expanded dengue syndrome

infection with atypical manifestations and required more supportive therapy.

\section{Discussion}

The most common atypical gastrointestinal presentations of dengue fever in the index study were hepatitis, fulminant hepatic failure, portal hypertension, acalculous cholecystis, appendicitis, acute pancreatitis, acute parotitis and febrile diarrhea.

Hepatitis was present in $11.4 \%$ of cases and among them two children developed fulminant hepatic failure. The etiology of hepatic dysfunction in dengue fever is usually due to direct cytopathic injury, unregulated host immune response, active viral replication, and hypoxia and tissue ischemia due to prolonged shock, hemorrhage and metabolic acidosis [2, 4-6].

Acute pancreatitis is a rare complication of dengue fever due to possible direct cytopathic effect of virus or an autoimmune response by molecular mimicry causing pancreatic outflow obstruction by resulting edema and ultrasound abdomen is characterized by enlarged pancreas and elevated serum amylase and lipase levels [7]. A child presented to the authors' hospital with severe dengue with features of acute pancreatitis and responded to symptomatic treatment.

Acute acalculous cholecystitis is equally rare in dengue fever. The main pathophysiological changes in dengue fever could be due to increased vascular permeability causing plasma leakage and serous effusion with high protein content which causes thickening of gall bladder wall [8]. The course of the disease is usually self-limiting and gall bladder wall thickness usually returns to normal with supportive care in majority of cases, even though isolated cases of gangrene and perforated gall bladder with peritonitis has been reported and surgical intervention is reserved only for children who have evidence of gangrene, perforation and diffuse peritonitis [2]. Gall bladder wall edema on ultrasound was a common associated finding of severe dengue in the index study similar 
to the previous studies [9]. Acute acalculous cholecystitis was seen in two children who improved symptomatically and were discharged.

In this study, febrile diarrhea was seen in $6.3 \%$ cases with severe dengue which is unusual and very few cases have been reported [10]. Bilateral parotid enlargement was present in 2 children in the index study while only one case has been reported in the past [2]. Acute appendicitis was seen in two cases with severe dengue which created a dilemma for surgeons but responded to conservative management, with very few cases being reported in the past [11].

Neurological manifestations of severe dengue include seizures, encephalopathy, meningitis, Gullian Barre syndrome, acute disseminated encephalomyelitis and transverse myelitis. It may occur because of factors like direct tissue invasion of virus (neurotropicity), cytokine mediated damage to the blood brain barrier, cerebral edema, intracranial hemorrhage, and secondary to cerebral hypoperfusion due to prolonged shock, renal failure, hepatic dysfunction and metabolic derangements like hyponatremia and hypoglycemia $[2,12]$. The neurological manifestations of dengue fever were first described in 1976 [13, 14]. Pancharoen and Thisyakorn, in their study, reported altered sensorium as the most common neurological finding followed by seizures and observed these findings in $75 \%$ of patients with dengue shock syndrome [15]. RigauPerez et al. and similar previous studies have reported a high proportion of impaired consciousness at presentation and during disease progression with multi-organ involvement and high mortality [16]. Dengue encephalopathy has been reported to occur in $0.5-17 \%$ patients with dengue [17-19]. In this study, impaired consciousness and seizures at admission were seen in six patients and among them there were four deaths $(66 \%)$; all had features of refractory shock and multiorgan failure, the most omnious sign for mortality with severe dengue.

The cardiac manifestations of dengue fever in the index study were myocarditis, paroxysmal supraventricular tachycardia, sinus bradycardia, pericardial effusion and ectopic ventricular beats. Majority of the patients had a spontaneous resolution and received symptomatic supportive treatment. The association of cardiac rhythm disturbances in dengue fever have been reportedly attributed to viral myocarditis during episodes of dengue hemorrhagic fever [20-22]. Pericardial involvement has also been attributed to dengue infection along with myocarditis [23].

Acute respiratory distress syndrome (ARDS) is one of the unusual and fatal complications of severe dengue infection. It is usually secondary to increased alveolar-capillary membrane permeability leading to interstitial and alveolar edema which leads to pulmonary dysfunction and is associated with high mortality rate [24]. Pulmonary hemorrhage with disseminated intravascular coagulopathy (DIC) is another fatal and unusual complication in severe dengue infection [25]. In the index study four children developed acute respiratory distress syndrome and pulmonary hemorrhage and had poor outcome as described in the previous studies [26].

Acute kidney injury was present in six cases in the present study. It is an unusual manifestation of dengue fever and mainly presents as shock induced acute tubular necrosis apart from other rare causes like multi-organ dysfunction and rhabdomyolysis. The role of immune complex in development of renal failure in dengue infection is still unclear [2,27]. Although hepatomegaly is among the WHO clinical criteria for dengue fever, splenomegaly and lymphadenopathy are not generally held to be a feature of dengue infection [1]. In the index study there was higher percentage of splenomegaly and lymphadenopathy compared to the previous studies [28]. Splenomegaly and lymphadenopathy signified the occurrence of dengue virus antigen in the lymphoreticular cells. Hemophagocytic syndrome is an unusual manifestation of dengue fever which was present in two cases with very few cases reported in the past [29].

Although myalgia is a common manifestation of dengue fever, myositis is unusual. The probable mechanism for myositis is the release of myotoxic cytokines, particularly tumor necrosis factor (TNF-alfa) thereby injuring the affected muscle [30]. These patients need to be detected promptly as there is a risk of progressing to renal failure if not identified in time [31]. In children presenting with myositis, dengue fever should be considered as an important differential diagnosis.

In the present study secondary infection was less than primary infection, but it was commonly associated with severe dengue. Wichmann et al. in their study showed that secondary infection was significantly associated with severe dengue in children. During secondary infection, T-cells become activated due to interactions with infected monocytes which induce plasma leakage by release of cascade of cytokines such as interferon-gamma, IL-2, and TNF-alfa [32].

Co-infections were seen in $17.4 \%$ cases and it is important that they be promptly recognized. Co-infections can modify the clinical presentation of dengue and result in missed or delayed diagnosis and treatment of dengue shock. Coexistence of malaria and dengue have been reported to be in the range of $20 \%$ to as high as $80 \%[2,3,33]$.

In the index study thrombocytopenia, bleeding and plasma leakage did not always correlate. Since majority of the cases were dengue fever with peripheral circulatory failure without bleeding, alternate case definitions used were Dengue fever associated with shock without bleeding (DSAS) and Dengue fever with bleeding without shock (DFB) and authors' experience suggests a need for revision of the existing case definitions [1]. Another unique observation was that majority of the cases of severe dengue presented with compensated shock without bleeding and responded to fluid therapy and only $6.3 \%$ developed complications. The most probable reasons being early detection of cases, availability of quick early 
diagnostic test like NS1 antigen detection, and timely hospitalization and increased awareness among the public. The bleeding manifestations also did not correlate well with platelet counts, and occurred in children with even normal platelet counts and platelet transfusion was given in children with severe dengue with severe thrombocytopenia. Coagulation profile was deranged in 13 cases $(5.1 \%)$ signifying the fact that factors other than thrombocytopenia like platelet dysfunction, consumption coagulopathy and endothelial dysfunction are responsible for bleeding in dengue fever [34].

The most common factors for atypical manifestations with complications were prolonged shock, bleeding, pain abdomen, lack of clinical improvement post defervescence, impaired consciousness, lethargy, restlessness and severe thrombocytopenia.

\section{Conclusions}

Atypical manifestations of dengue fever are more common than actually reported. However, the awareness is lacking among the health care personnels especially at primary health centers from where these cases are often referred. There is a need to update the health care personnels and community at various forums, about the various atypical manifestations of dengue for prompt recognition and management. Impaired consciousness at the time of admission should be considered as the most omnious atypical manifestation of severe dengue infection.

Acknowledgments The authors would like to thank Dr. Vijayalalakshmi Sivapurapu, intensivist for giving critical input to the manuscript.

Contributions SP was involved in doing the study, data collection and analysis, literature search, analyzing and drafting the manuscript. MT was involved in data analysis, statistical analysis and drafting the manuscript. BK was involved in data collection, analysis, literature search and drafting of the manuscript. SP was involved in critical review and finalizing the manuscript and will act as the guarantor of the paper.

Conflict of Interest None.

Source of Funding None.

\section{References}

1. World Health Organization, Regional Office for South-East Asia. Comprehensive guidelines for prevention and control of dengue and dengue haemorrhagic fever, Revised and expanded edition. WHO-SEARO 2011. (SEARO Technical Publication Series No $60)$.
2. Gulati S, Maheshwari A. Atypical manifestations of dengue. Tropical Med Int Health. 2007;12:1087-95.

3. Balasubramanuim S, Ramachandran B, Amperayani S. Dengue viral infection in children: a perspective. Arch Dis Child. 2012;97:907-12.

4. Kumar R, Tripathi P, Tripathi S, Konodia A, Venkatesh V. Prevalence of dengue infection in north Indian children with acute hepatic failure. Ann Hepatol. 2008;7:59-62.

5. Parkash O, Almas A, Jafri SM, Hamid S, Akhtar J, Alishah H. Severity of acute hepatitis and its outcome in patients with dengue fever in a tertiary care hospital Karachi, Pakistan (south Asia). BMC Gastroenterol. 2010;10:43.

6. Chhina RS, Goyal O, Chinna DK, Goyal P, Kumar R, Puri S. Liver function tests in patients with dengue viral infection. Dengue Bull. 2008;32:110-7.

7. Gonzalez-Fontal GR, Henao-Martinez AF. Dengue hemorrhagic fever complicated by pancreatitis. Braz J Infect Dis. 2011;15:490-2.

8. KoH FH, Misli H, Chong VH. Acute acalculous cholecystitis secondary to dengue fever. Brunei Int Med J. 2011;7:45-9.

9. Colbert JA, Gordon A, Roxelin R, et al. Ultrasound measurement of gall bladder wall thickening as a diagnostic test and prognostic indicator for severe dengue infection in pediatric patients. Pediatr Infect Dis J. 2007;26:850-2.

10. Helbok R, Dent W, Gattringer K, Innerebner M, Schmutzhard E. Imported dengue fever presenting with febrile diarrhea: report of two cases. Wein Klin Wochenschr. 2004;116:S58-60.

11. McFarlane ME, Plummer JM, Leake PA, et al. Dengue fever mimicking acute appendicitis: a case report. Int J Surg Case Rep. 2013;4:1032-4.

12. Verma R, Sharma P, Garg RK, Atam V, Singh MK, Mehrotra HS. Neurological complications of dengue fever: experience from a tertiary centre of north India. Ann Indian Acad Neurol. 2011;14: 272-8.

13. Misra UK, Kalita J, Syam UK, Dhole TN. Neurological manifestations of dengue virus infection. J Neurol Sci. 2006;244:117-22.

14. Solomon T, Dung MN, Vaughn DW, et al. Neurological manifestations of dengue infection. Lancet. 2000;355:1053-9.

15. Pancharoen $\mathrm{C}$, Thisyakorn U. Neurological manifestations in dengue patients. Southeast Asian J Trop Med Public Health. 2001;32: 341-5.

16. Garcia-Rivera EJ, Rigau-Perez JG. Encephalitis and dengue. Lancet. 2002;360:261.

17. Murthy J Neurological complications of dengue infection. Neurol India. 2010;58:581-4.

18. Kamath SR, Ranjit S. Clinical features complications and atypical manifestations of children with severe forms of dengue hemorrhagic fever in south India. Indian J Pediatr. 2006;73:889-95.

19. Kumar R, Tripathi S, Tambe JJ, Arora V, Srivastava A, Nag VL. Dengue encephalopathy in children in northern India: clinical features and comparison with non-dengue. J Neurol Sci. 2008;269:41-8.

20. Lee IK, Lee WH, Liu JW, Yank KD. Acute myocarditis in dengue hemorrhagic fever: a case report and review of cardiac complications in dengue-affected patients. Int J Infect Dis. 2010;14:e919-22.

21. Promphan W, Sopontamarak S, Pruekprasert P, Kajornwatttanakul W, Kongapattanayothin A. Dengue myocarditis. Southeast Asian J Trop Med Public Health. 2004;35:611-3.

22. Khongphathanayothin A, Suesaowalak M, Muangmingsook S, Bhattarakosol P, Pancharoen C. Hemodynamic profiles of patients with dengue hemorrhagic fever during toxic stage: an echocardiographic study. Intensive Care Med. 2003;29:570-4.

23. Pelupessy JM, Allo ER, Jota S. Pericardial effusion in dengue haemorrhagic fever. Paediatr Indones. 1989;29:72-5.

24. Lum LC, Thong MK, Cheah YK, Lam SK. Dengue associated adult respiratory distress syndrome. Ann Trop Paediatr. 1995;15: 335-9. 
25. Liam CK, Yap BH, Lam SK. Dengue fever complicated by pulmonary haemorrhage manifesting as hemoptysis. J Trop Med Hyg. 1993;96:197-200.

26. Dhooria GS, Bhat D, Bains HS. Clinical profile and outcome in children of dengue hemorrhagic fever in north India. Iran J Pediatr. 2008; 18:222-8.

27. Wiwanitkit V Acute renal failure in the fatal cases of dengue hemorrhagic fever, a summary in Thai death cases. Ren Fail. 2005;27: 647.

28. Faridi MM, Agarwal A, Kumar M, Sarafrazul A. Clinical and biological profile of dengue hemorrhagic fever in children in Delhi. Trop Dr. 2008;38:28-30.

29. Ramachandran B, Balasubrmanian S, Abhishek N, Ravikumar KG, Ramanan AV. Profile of hemophagocytosis in children in a tertiary care hospital in India. Indian Pediatr. 2011;48:31-5.
30. Kalita J, Misra UK, Maurya PK, Shankar SK, Mahadevan A. Qualitative electromyography in dengue-associated muscle dysfunction. J Clin Neurophysiol. 2012;29:468-71.

31. Davis JS, Bourke P. Rhabdomyolysis associated with dengue virus infection. Clin Infect Dis. 2004;38:e109-11.

32. Wichmann $\mathrm{O}$, Hongsiriwon $\mathrm{S}$, Bowonwatanuwong $\mathrm{C}$, et al. Risk factors and clinical features associated with severe dengue infection in adults and children during the 2001 epidemic in Chonburi, Thailand. Trop Med Int Health. 2004;9:1022-9.

33. Pancharoen $\mathrm{C}$, Thisyakorn U. Coinfections with dengue patients. Pediatr Infect Dis J. 1998;17:81-2.

34. Mairuhu AT, Macgillavry MR, Setiati TE. Is clinical outcome of dengue-virus infections influenced by coagulation and fibrinolysis? A critical review of the evidence. Lancet Infect Dis. 2003;3:33-41. 